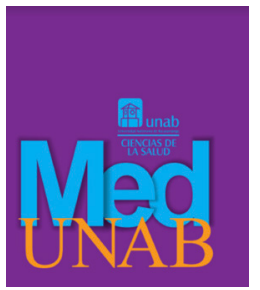

REVISTA DE LA FACULTAD

DE CIENCIAS DE LA SALUD

\title{
Estrategias de prevención y control de las infecciones en pacientes oncológicos.
}

Strategies to prevent and control infections in cancer patients.

Estratégias de prevenção e controle das infecções em pacientes oncológicos.

Adriana Patricia Bonilla-Marciales, Enf., Esp., MSc. ${ }^{1}$ (D), Wilmer Orlando Chávez-Cañas, Enf., Esp. ${ }^{2}$ (D) Ruby Alexandra Hernández-Mogollón, Enf. ${ }^{3 \mathbb{D}}$, Nathaly Andrea Ramón-Jaimes. ${ }^{4}$ (D)

1. Enfermera, Especialista en Docencia Universitaria, Magíster en Enfermería y en Docencia, Universidad Autónoma de Bucaramanga, Bucaramanga, Santander, Colombia.

2. Enfermero, Especialista en Enfermería Oncológica, candidato a Magíster en Ciencias de la Enfermería con énfasis en Hematología-Oncología, Hospital Internacional de Colombia, Floridablanca, Santander, Colombia.

3. Enfermera, ModerneTalen PVCO, Meeuwen (Oudsbergen), Limburg, Bélgica.

4. Estudiante de Enfermería, Universidad Autónoma de Bucaramanga, Bucaramanga, Santander, Colombia.

Correspondencia. Adriana Patricia Bonilla Marciales, Enfermera profesional, Especialista en Docencia Universitaria, Magíster en Enfermería y en Docencia. Universidad Autónoma de Bucaramanga, Campus El Bosque. Calle 157 No. 14 - 55. Cañaveral Parque. Floridablanca, Santander. Email. abonilla712@unab.edu.co

Cómo citar: Bonilla-Marciales AP, Chávez-Cañas WO, Hernández-Mogollón RA, Ramón-Jaimes NA. Estrategias de prevención y control de las infecciones en pacientes oncológicos. MedUNAB. 2019;22(3):356-368. doi: $10.29375 / 01237047.3376$

\section{INFORMACIÓN DEL ARTÍCULO:}

Artículo recibido: 17 de mayo de 2019

Artículo aceptado: 23 de septiembre de 2019

Doi: https://doi.org/10.29375/01237047.3376

\section{RESUMEN}

Introducción. El cáncer es uno de los problemas de salud pública más graves a nivel mundial. A pesar de los tratamientos y avances tecnológicos, continúan presentándose elevadas cifras de infecciones en los pacientes oncológicos. $\mathrm{La}$ enfermería cumple un papel fundamental al prevenir el riesgo de infección que conllevan las diferentes medidas terapéuticas utilizadas. El control de las Infecciones 
Asociadas a la Atención en Salud (IAAS) es imprescindible, dado que se considera la labor del cuidado permanente de la enfermera. El objetivo del presente artículo es el de realizar una búsqueda de literatura sobre las principales estrategias de control para la prevención de infecciones en pacientes oncológicos.

División de los temas tratados. Se realizó una búsqueda en las bases de datos Medline, Pubmed, Ebsco, Scielo y Clinicalkey entre el 2011 y el 2018 con el propósito de encontrar información sobre la prevención de infecciones en pacientes oncológicos. Se expone la definición, epidemiología y las diferentes formas de control en la prevención de infecciones en pacientes oncológicos, clasificando tales medidas preventivas en generales y específicas.

Conclusiones. La literatura científica ofrece una gran variedad de recomendaciones para la prevención de infecciones en pacientes oncológicos. Se pueden minimizar los riesgos a través de la implementación de estrategias que demuestren evidencia científica como es la nutrición y las medidas de higiene.

Palabras clave:

Control de infecciones; Enfermería; Pacientes; Oncología; Prevención de enfermedades.

\section{ABSTRACT}

Introduction. Cancer is one of the most serious global public health problems. Despite the treatments and technological advances, cancer patients continue to present high rates of infection. Nursing plays an essential role in preventing the risk of infection brought by the different therapeutic measures used. Control of Health Care-Associated Infections (HAIs) is essential, because it is considered part of the ongoing care work of nurses. The aim of this article is to conduct a literary review of the main control strategies for the prevention of infections in cancer patients.

Division of Covered Topics. A search was conducted in the Medline, Pubmed, Ebsco, Scielo and Clinicalkey databases between 2011 and 2018 to find information about the prevention of infections in cancer patients. The definition, epidemiology and different control methods in the prevention of infections in cancer patients are described, classifying these preventive measures as general or specific.

Conclusions. The scientific literature offers a great variety of recommendations for the prevention of infections in cancer patients. The risks can be minimized through the implementation of strategies supported by scientific evidence, such as nutrition and hygiene measures.

Keywords:

Infection control; Nursing; Patients; Oncology; Disease prevention.

\section{RESUMO}

Introdução. O câncer é um dos mais graves problemas de saúde pública em todo o mundo. Apesar dos tratamentos e avanços tecnológicos, uns grandes números de infecções em pacientes oncológicos continuam a ocorrer. A enfermagem desempenha papel fundamental na prevenção dos riscos de infecção associados às diferentes medidas terapêuticas utilizadas. O controle das Infecções Associadas aos Cuidados de Saúde (IACS) é essencial, uma vez que é considerada a função de cuidado permanente do enfermeiro. O objetivo deste artigo é realizar uma pesquisa bibliográfica sobre as principais estratégias de controle para a prevenção de infecções em pacientes oncológicos.

Divisão dos tópicos discutidos. Foram realizadas buscas nas bases de dados Medline, Pubmed, EBSCO, ScIELO e Clinical Key no período de 2011 a 2018 com o objetivo de encontrar informações sobre a prevenção de infecções em pacientes oncológicos. Apresentam-se a definição, epidemiologia e as diferentes formas de controle na 
prevenção de infecções em pacientes oncológicos, classificando essas medidas preventivas em geral e específica.

Conclusão. A literatura científica oferece uma ampla variedade de recomendações para a prevenção de infecções em pacientes oncológicos. Os riscos podem ser minimizados através da implementação de estratégias que demonstram evidência científica como a nutrição e as medidas de higiene.

Palavras-chave:

Controle de Infecções; Enfermagem; Pacientes; Oncologia; Prevenção de Doenças.

\section{Introducción}

Cáncer es un término que se usa para denominar las enfermedades en las que ciertas células anormales se dividen sin control y pueden invadir otros tejidos. Estas células pueden diseminarse a otras partes del cuerpo por el sistema sanguíneo y por el sistema linfático (1). La transformación de células normales en células tumorales es un proceso de varias etapas que consiste en la progresión de una lesión precancerosa a un tumor maligno. Su incidencia aumenta exponencialmente con la edad, debido a la acumulación de factores de riesgo y a la disminución, natural por la edad, de la eficacia de los mecanismos de reparación celular (2).

Al convertirse esta enfermedad en la primera causa de mortalidad, con el paso de los años se ha venido incrementando la demanda de atención médica especializada, lográndose avances a nivel asistencial y terapéutico, que ha significado una mejora en el pronóstico de los pacientes oncológicos. Sin embargo, las intervenciones traen consigo diversos factores de riesgo que pueden producir el aumento de infecciones, que en tanto complicaciones debilitan el estado de salud de la persona (3). Debido a que el paciente oncológico posee mayor riesgo de infecciones por estar expuesto a múltiples factores de riesgo, el personal de enfermería juega un papel importante a la hora de vigilar, identificar y prevenir las causas más comunes del desarrollo de infecciones, así como de utilizar diferentes métodos para el control de estas (4).

La atención del paciente oncológico inicia con el primer contacto que tiene con los servicios de salud a raíz de los síntomas de la enfermedad o los resultados de pruebas de tamización. Esto indica que la atención no solo interviene el manejo de la patología sino que además exige una adecuada comunicación entre quienes inician el proceso de cuidado oncológico, en todos los aspectos que se tengan que abarcar durante el proceso (5). Los tratamientos en el paciente oncológico tienen como objetivo la destrucción de las células cancerígenas. Desafortunadamente, las terapias utilizadas afectan las células de manera sistémica, provocando una alteración del sistema inmunológico, aumentando la probabilidad de contraer infecciones $(4,5)$.

"La hospitalización aumenta el riesgo de desarrollar infecciones asociadas a la atención en salud, ya que existen factores como tratamientos invasivos, cirugía, radioterapia, quimioterapia, multirresistencia a los antimicrobianos secundarios al uso inadecuado de los antimicrobianos de amplio espectro, la neutropenia y la condición de base del paciente con cáncer" (5).

Los programas de control de infecciones incluyen precauciones estándar, precauciones de aislamiento, saneamiento ambiental y vigilancia. Estas precauciones estándar se deben tomar con todos los pacientes hospitalizados para reducir el riesgo de transmisión de infección entre pacientes y trabajadores de la salud, se conozca o no la presencia de un agente infeccioso (7).

Por lo expuesto anteriormente, se realizó una búsqueda de artículos científicos y guías publicados en el periodo entre el 2011 y el 2018 que aborden las principales medidas de control para la prevención de infecciones en pacientes oncológicos.

\section{Epidemiología}

El cáncer es una de las principales causas de morbilidad y mortalidad en el mundo. En 2012 se registraron alrededor de 14 millones de nuevos casos y se prevé que el número de nuevos casos aumente aproximadamente un $70 \%$ en 20 años. Es la segunda causa de muerte en el mundo: en el año 2015 ocasionó 8.8 millones de defunciones y una de cada seis defunciones en el mundo se debe a esta enfermedad (6).

La detección de cáncer en una fase avanzada junto con la falta de diagnóstico y tratamiento son problemas frecuentes. En 2015, solo el 35\% de los países de ingresos bajos informaron que la sanidad pública contaba con servicios de patología para atender a la población en general. Más del $90 \%$ de los países de ingresos altos ofrecen tratamiento a los enfermos 
oncológicos, mientras que en los países de ingresos bajos este porcentaje es inferior al 30\%. Lo anterior refleja el impacto económico del cáncer como una problemática sustancial y en aumento, pues según las estimaciones, el costo total atribuible a la enfermedad en 2010 ascendió a USD 1.16 billones (6).

El panorama en América no está lejos de la realidad mundial: en el 2012 causó 1.3 millones de muertes, un 47\% de las cuales ocurrieron en América Latina y el Caribe. Se espera que la mortalidad por cáncer en las Américas aumente hasta 2.1 millones en el 2030 (7). En Colombia, el cáncer es considerado un problema importante de salud pública. La morbilidad por esta causa ha ido aumentando en los últimos años. Para el 2012 se reportaron cerca de 71,000 casos nuevos y 38,000 muertes, lo que significa que diariamente 195 personas son diagnosticadas y 104 más mueren por esta enfermedad (8).

Las Infecciones Asociadas a laAtención en Salud(IAAS) son aquellas que el paciente adquiere mientras recibe tratamiento para alguna condición médica o quirúrgica y la cual no se había manifestado ni estaba en período de incubación en el momento del ingreso a la institución (9). Se producen por numerosas causas, relacionadas tanto con los sistemas y procesos de la prestación de la atención sanitaria, como con comportamientos individuales (10). Dentro de las diferentes herramientas para la gestión y control se encuentra la prevención, que permite identificar factores de riesgo e implementar acciones para eliminar o disminuir los riesgos de contraer y transmitir infecciones entre pacientes, personal de salud y público en general (11).

Las complicaciones infecciosas desencadenan en alteraciones del sistema inmune, cosa que aumenta los riesgos asociados a la enfermedad. En los pacientes oncológicos las infecciones más comunes son infecciones del catéter venoso $(5 \%-26 \%)$, infecciones del tracto respiratorio $(0.4 \%-35 \%)$, infecciones del tracto digestivo ( $30 \%$ en presencia de fiebre), mucositis y esofagitis $(40 \%-76 \%)$, infecciones intraabdominales $(15 \%-50 \%)$, infecciones perirectales, infecciones genitourinarias e infecciones del sistema nervioso central (SNC) (11).

Velásquez y colaboradores reportan que "los factores de riesgo más frecuentes a los que estuvieron expuestos los pacientes que desarrollaron una IAAS fueron: sonda vesical $52 \%$, catéter periférico $46 \%$, catéter central y estomas $34 \%$, nebulizaciones $32 \%$, hemotransfusión $30 \%$, alimentación parenteral $26 \%$, sonda nasogástrica $24 \%$, oxigenoterapia $22 \%$, cirugía previa $16 \%$, ventilación mecánica $14 \%$, drenaje Penrose $12 \%$, hospitalización previa $10 \%$, quimioterapia, catéter Mahurkar 4\%" (12).

Enfermería es una profesión esencial para el control de las IAAS, gracias al trabajo que realiza de vigilancia epidemiológica, de entrenamiento y de supervisión de la ejecución de rutinas (13), logrando así el cumplimiento con las prácticas de atención a los pacientes para el control de dichas infecciones durante su estancia hospitalaria (14).

\section{Medidas de prevención para infecciones en pacientes oncológicos}

Teniendo en cuenta la información encontrada en la revisión, las recomendaciones pueden clasificarse en medidas de control generales y específicas para la prevención de infecciones en el paciente oncológico (Tablas 1 y 2).

\section{Medidas generales de control}

\section{Recomendación 1.1 - lavado de manos}

Uno de los principales mecanismos de trasmisión de microorganismos en el hospital es el contacto con las manos contaminadas del personal de salud. Los pacientes de oncología son pacientes críticos con alteraciones importantes en su sistema inmune (5). La higiene de manos es la medida más importante para reducir la transmisión de microorganismos de una persona a otra o de una parte del cuerpo a otra en el mismo paciente. Tales medidas son sencillas y de fácil aprendizaje y realización. Se componen del lavado o descontaminación de manos, que llamaremos "higiene de manos" (5). Este concepto engloba el lavado de arrastre con agua junto con un agente antiséptico y la desinfección con soluciones alcohólicas. La utilización de soluciones alcohólicas supuso una revolución en la higiene de manos al permitir una mayor accesibilidad a los productos, por la rapidez y por no requerir secado posterior. La fórmula recomendada por la Organización Mundial de la Salud (OMS) es de 75\% isopropanol u 80\% etanol (5).

Indicaciones para el lavado de manos y la descontaminación:

- Lavado de manos con agua y jabón

- Al iniciar y terminar la jornada laboral.

- Manos visiblemente manchadas o sucias.

- Manos contaminadas con sangre o fluidos corporales. 
Tabla 1. Clasificación de las medidas generales y específicas de prevención de infecciones.

\begin{tabular}{lll} 
MEDIDAS GENERALES DE CONTROL & NIVEL DE \\
\hline N. & RECOMENDACIÓN & Ia \\
\hline 1.1 & $\begin{array}{l}\text { LAVADO DE MANOS: Se recomienda el lavado de manos, en } \\
\text { los cinco momentos con agua y un agente antiséptico/ jabón según } \\
\text { corresponda. }\end{array}$ & \\
\hline 1.2 & $\begin{array}{l}\text { AISLAMIENTO: Se define según el tipo de aislamiento: } \\
\text { Respiratorio: Habitación individual, uso de mascarillas, limitar salidas } \\
\text { de la habitación, cuarentena de la habitación de seis a doce horas. }\end{array}$ & Ib \\
& $\begin{array}{l}\text { GOTAS: Habitación individual, bata, mascarilla quirúrgica, limitar } \\
\text { salidas del paciente } \\
\text { CONTACTO: Habitación individual, dos contenedores, limpieza diaria } \\
\text { habitual y limpieza terminal a la salida del paciente. }\end{array}$ & \\
\hline 1.3 & $\begin{array}{l}\text { DESINFECCIÓN DE LAS ÁREAS: Limpieza con productos } \\
\text { que garanticen la eliminación de patógenos: soluciones de lejía, } \\
\text { desinfección exhaustiva al alta del paciente. }\end{array}$ & Ib \\
\hline 1.4 & $\begin{array}{l}\text { CALIDAD DEL AGUA: Asegurar el control y viabilidad del } \\
\text { agua a través de las diferentes tecnologías existentes para evitar la } \\
\text { colonización de microorganismos. }\end{array}$ & IIb \\
\hline BIOSEGURIDAD: Se recomienda el uso de mascarilla y guantes \\
constantemente y bata según el tipo de contacto.
\end{tabular}

$2.1 \quad$ NUTRICIÓN: Dieta Neutropénica: Es controvertida, a falta de

$\mathrm{Ib}$ evidencia se recomienda la restricción de alimentos que entrañen un riesgo.

\begin{tabular}{lll}
\hline 2.2 & FAMILIA: Se recomienda integrar a la familia en el plan de cuidados. & $\mathrm{IIb}$ \\
\hline 2.3 & $\begin{array}{l}\text { INTERVENCIÓN DE CONTROL: Se recomienda realizar biometrías } \\
\text { de control para detectar precozmente la infección en el paciente. }\end{array}$ & $\mathrm{IIb}$ \\
\hline 2.4 & $\begin{array}{l}\text { AMBIENTAL: No se recomienda tener flores en la unidad; los equipos } \\
\text { en la habitación e individuales, no mover los equipos de habitación y } \\
\text { de ser necesario desinfectarlos previamente. }\end{array}$ & $\mathrm{Ib}$ \\
\hline 2.5 & $\begin{array}{l}\text { EDUCACIÓN: Se recomienda suministrar información actualizada } \\
\text { y capacitación al personal de enfermería sobre los riesgos y } \\
\text { cómo disminuir o prevenir los factores de riesgo de infección con } \\
\text { evaluaciones periódicas. }\end{array}$ & $\mathrm{Ia}$ \\
\hline 2.6 & $\begin{array}{l}\text { VACUNACIÓN: Se recomienda su uso para disminuir el riesgo de } \\
\text { infección. }\end{array}$ & $\mathrm{Ib}$ \\
\hline 2.7 & CUIDADOS DEL CATÉTER VENOSO CENTRAL & $\mathrm{Ia}$ \\
\hline 2.8 & CUIDADOS DEL CATÉTER URINARIO & $\mathrm{Ib}$
\end{tabular}

Fuente: Elaborado por los autores. 
Tabla 2. Indicaciones de aplicación de las precauciones estándar.

\begin{tabular}{lllll} 
PROCEDIMIENTO & $\begin{array}{l}\text { DESCONTAMINACIÓN } \\
\text { MANOS }\end{array}$ & GUANTES & BATA & MASCARILLA \\
\hline No contacto & NO & NO & NO & NO \\
\hline $\begin{array}{l}\text { Contacto con piel } \\
\text { intacta o ropa no } \\
\text { manchada }\end{array}$ & ANTES Y DESPUÉS & NO & NO & $\begin{array}{l}\text { NO - Excepto } \\
\text { curación }\end{array}$ \\
\hline $\begin{array}{l}\text { Contacto o } \\
\text { posibilidad con piel } \\
\text { no intacta, mucosas, } \\
\text { fluidos o secreciones }\end{array}$ & ANTES Y DESPUÉS & $\begin{array}{l}\text { Sí - Cambiar entre } \\
\text { pacientes y zonas }\end{array}$ & $\begin{array}{l}\text { NO - Excepto } \\
\text { curación }\end{array}$ & $\begin{array}{l}\text { NO -Excepto } \\
\text { curación }\end{array}$ \\
\hline $\begin{array}{l}\text { Secreciones } \\
\text { respiratorias }\end{array}$ & ANTES Y DESPUÉS & $\begin{array}{l}\text { SÍ - Cambiar } \\
\text { entre pacientes y } \\
\text { zonas }\end{array}$ & SÍ & SI \\
\hline
\end{tabular}

Fuente: Carmen Lupióna; Luis Eduardo López-Cortésa, Jesús Rodríguez-Baño; Medidas de prevención de la transmisión de microorganismos entre pacientes hospitalizados. Higiene de manos; Enferm Infecc Microbiol Clin 2014; 32:603-9 - DOI: 10.1016/j.eimc.2014.02.003)(26).

- Antes y después de comer, preparar, repartir o servir comida.

- Después de ir al baño.

- Después de estornudar, sonarse, etc.

- Después de tener contacto físico con pacientes que presenten infección por Clostridium difficile (7).

- Descontaminación de manos

(Puede realizarse con solución alcohólica, o por lavado de manos con agua y jabón antiséptico)

- Antes del contacto directo con el paciente.

- Antes de la colocación de los guantes.

- Después de tocar la piel intacta de un paciente (tomar pulso o la tensión arterial, levantar al paciente, etc.).

- Al pasar las manos de un punto corporal sucio a otro limpio durante el cuidado del paciente.

- Después de contactar con líquidos orgánicos o excreciones, membranas, mucosas, piel no intacta y vendajes de heridas, así no estén visiblemente manchadas.

- Tras la retirada de los guantes.

- Tras utilizar objetos situados cerca del paciente: equipos médicos, cuñas, bombas de perfusión, ventiladores, etc. (7).

\section{Recomendación 1.2 - aislamiento}

Hay tres categorías de aislamiento que reflejan los principales modos de transmisión del microorganismo en las configuraciones nosocomiales: contacto, gotas, y aerosoles. Las recomendaciones varían según el tipo de aislamiento:

\section{- Aerosol/respiratorio}

- Habitación individual: $\mathrm{Si}$ es posible, con presión negativa con 6-12 intercambios de aire por hora y salida de aire directamente al exterior o filtrado a través de un filtro de alta eficacia. La puerta de la habitación debe permanecer siempre cerrada para preservar estas condiciones.

- Para entrar en la habitación, esté o no el paciente, deben usarse respiradores (mascarillas) de alta filtración inspiratoria (filtro HEPA).

- Las salidas del paciente deben limitarse a las necesarias. En estos casos debe colocarse una mascarilla quirúrgica al paciente $\left(\mathrm{N} .^{\circ} 75\right)$

- Tras el alta, la habitación debe permanecer cerrada $6 \mathrm{~h}$ antes de ser ocupada de nuevo. Si no existe un sistema de alto recambio de aire, el tiempo debe ser de $12 \mathrm{~h}$ al menos (7).

- Gotas

- Habitación individual o en su defecto separación de otros pacientes de mínimo 1 metro de distancia. 
- Uso de bata para el contacto directo. La bata debe estar en la habitación del paciente.

- Uso de mascarilla quirúrgica.

- Limitación de salidas de la habitación (7).

- Contacto

- Habitación individual o en su defecto pacientes con el mismo patógeno en la misma habitación.

- Dos contenedores: uno para ropa y otro para desechos.

- Uso de bata no estéril y guantes. Al terminar, desechar en el contenedor.

- Utilizar mascarilla cuando se realice aspiración de secreciones (7).

\section{Recomendación 1.3 - desinfección de las áreas}

Las superficies deben considerarse uno de los reservorios que más pueden albergar patógenos. Un huésped susceptible es uno de los componentes que subraya la influencia del entorno en las infecciones asociadas al cuidado de la salud, debido a los patógenos oportunistas en fómites, aire y agua. Como resultado de los avances hechos en la tecnología y tratamientos médicos, se aumenta el riesgo y los pacientes se vuelven susceptibles en el curso del tratamiento. Por lo tanto, enfrentan un riesgo adicional de adquirir infecciones oportunistas en las instituciones de salud. El medio ambiente inanimado presente en toda institución de salud guarda una íntima relación con las infecciones asociadas al cuidado médico, y puede contribuir a casos esporádicos o a brotes de una enfermedad en instituciones, pues constituye focos de contagio y transmisión de gérmenes por vehículo común, por el aire y por vectores (15).

\section{Recomendación 1.3.1 - limpieza de superficies}

- Contacto mínimo

- Desinfección periódica de áreas horizontales

o Cuando se presenten salpicaduras o suciedad alta del paciente (15).

- Contacto frecuente

- Desinfección frecuente de perillas, camas, interruptores, pared del baño (15).

Recomendación 1.3.2 - Recomendaciones generales

- Desempolvar diariamente y en húmedo las superficies horizontales utilizando paños de limpieza humedecidos con detergente desinfectante.

- Evitar el contacto del paciente con el detergente desinfectante.
- Evitar el uso de equipos de aseo que produzcan vapores o aerosoles.

- Utilizar soluciones recién preparadas de detergentes o desinfectantes.

- La contaminación bacteriana y por hongos de los filtros en el equipo de limpieza es inevitable, y estos elementos deben limpiarse periódicamente o reemplazarse de acuerdo con las instrucciones del fabricante y de los miembros del comité de infecciones intrahospitalarias de la institución.

- Las puertas de las habitaciones de los pacientes en general y de los inmunosuprimidos deben cerrarse cuando se estén limpiando áreas vecinas.

- No utilice alcohol para desinfectar superficies ambientales grandes.

- Evite los métodos de limpieza de grandes superficies que produzcan aerosoles o dispersen polvo en las áreas de cuidado del paciente (15).

\section{Recomendación 1.4-agua}

La buena calidad del agua es un prerrequisito en el óptimo cuidado del paciente y en el bienestar de los visitantes y el hospital. Con relación a la calidad del agua se recomienda utilizar métodos de medición de cloro y tecnologías de filtración de membrana para microbiología del agua, específicamente para los siguientes casos:

- Pseudomona aeruginosa en el agua, para lo que se recomienda asegurar una cloración óptima del suministro de agua.

- Alto recuento de colonias de Clostridium en el suministro de botellas agua potable, a lo que recomienda el cambio periódico de corto plazo y la dispensación del agua (16).

\section{Recomendación 1.5 - bioseguridad}

Se define como el conjunto de medidas preventivas destinadas a mantener el control de factores de riesgo laborales procedentes de agentes biológicos, físicos o químicos, logrando la prevención de impactos nocivos, asegurando que el desarrollo o producto final de dichos procedimientos no atenten contra la salud y seguridad de trabajadores de la salud, pacientes, visitantes y el medio ambiente (17). Para esto se recomienda:

- Uso de barreras (guantes, bata, mascarilla, etc.)

- El uso de guantes juega un papel importante en la reducción de los riesgos de transmisión de microorganismos. Proporcionan una 
barrera protectora para las manos del personal de salud de material contaminado y reducen la posibilidad de colonización de la piel del trabajador de microorganismos de un paciente que está colonizado o infectado con organismos patógenos.

- La bata y gafas protectoras se deben utilizar en situaciones en las que la exposición a las secreciones del paciente o la sangre sea posible o probable.

- Las máscaras tienen propósitos como proteger no solo al personal de salud sino también a los pacientes inmunosuprimidos, además de limitar la propagación de las secreciones respiratorias infecciosas de los pacientes que tosen.

- Eliminación segura delos elementos cortopunzantes en recipientes para tal fin

- Ubicación de las sábanas sucias y materiales contaminados con sangre en bolsas impermeablesEl desecho de heces y orina en el sanitario son medidas fundamentales en el control de infecciones.

Para llevar a cabo las medidas mencionadas se cuenta, en las instituciones prestadoras de salud, con protocolos, manuales y guías que cada institución adapta, dependiendo de las necesidades y los controles que desea implementar (18-20)

\section{Medidas específicas de control}

\section{Recomendación 2.1 - dieta}

"La desnutrición es frecuente en pacientes con cáncer, y afecta negativamente a la evolución de la enfermedad. Algunos estudios demuestran un $27 \%$ de desnutrición o riesgo nutricional en pacientes sometidos a trasplante de progenitores hematopoyéticos (TPH). A ello contribuye la intensidad del tratamiento administrado, que incluye, en algunos casos, procedimientos complejos como el TPH, lo que supone un elevado estrés metabólico. Además, los efectos secundarios de los tratamientos pueden contribuir en mayor o menor medida a la desnutrición del paciente especialmente los relacionados con el tracto gastrointestinal, pudiendo disminuir y/o dificultar la ingesta, digestión y absorción de nutrientes" (21).

Los objetivos del soporte nutricional en el paciente oncohematológico incluyen el mantenimiento de un buen estado nutricional; la prevención y el tratamiento de complicaciones derivadas de los fármacos utilizados, o de la propia enfermedad, que tengan un impacto sobre el mismo; y, en definitiva, mejorar la calidad de vida del paciente (21).

La desnutrición que aparece en el paciente oncohematológico es, con frecuencia, calórica y proteica. El uso de dietas de baja carga bacteriológica puede disminuir la incidencia de infecciones, disminuyendo la exposición de agentes bacterianos durante el periodo de neutropenia. Es difícil establecer comparaciones debido a la amplia variabilidad en las restricciones dietéticas; es necesaria la realización de más estudios en esta área. Hasta entonces, la indicación de restricciones dietéticas en la compra, almacenamiento, manipulación y preparación de ciertos alimentos durante el período de neutropenia es indispensable.

Las recomendaciones básicas en la dieta de baja carga bacteriológica incluyen:

- Utilizar normas de higiene y manipulación de alimentos para evitar la contaminación.

- Evitar el consumo de carnes, pescados o huevos crudos.

- Utilizar alimentos pasteurizados, envasados y cocinados siempre que sea posible.

- Evitar vegetales crudos (22-24).

\section{Recomendación 2.2 - familia}

"Las personas a cargo del paciente desempeñan una función importante en la toma de decisiones acerca del tratamiento y la atención. La familia a menudo entra como representante de los intereses del paciente y está encargada de adoptar las decisiones principales a pedido del paciente. La propiedad con que la persona a cargo de un paciente cumpla esa función puede depender de su relación anterior con el paciente y el grado de acuerdo entre ellos" (25).

La familia posee un papel fundamental para el cuidado y la potenciación del autocuidado en el paciente, siendo un pilar fundamental tanto para el paciente a nivel psicológico, emocional, social y físico, como para el personal de enfermería, ya que representa el soporte que previene complicaciones antes, durante y después de la atención en salud del paciente $(26,30)$.

\section{Recomendación 2.3 -intervención de control}

Continuando con la línea de prevención, se hace necesaria la caracterización de aquellos pacientes en los que el riesgo de infección es alto, para realizar biometrías de control para la prevención y diagnóstico 
precoz de la infección en esta población tan vulnerable, prestando especial atención en los pacientes con catéter venoso central (CVC), catéter urinario y pacientes inmunosuprimidos $(27,31)$. Se recomienda la toma de hemogramas con vigilancia en la línea blanca y parcial de orina con urocultivo, especialmente si el paciente tiene un catéter urinario permanente o presenta sintomatología de infección urinaria o sistémica $(31,32)$. Se destaca el uso de la clorhexidina a través de los baños para realizar la descolonización, disminuyendo así el riesgo de infección. Si bien la propuesta es atrayente, no hay un consenso con respecto a las características que debe reunir el paciente para tener aplicabilidad $(28,33)$.

\section{Recomendación 2.4 - vacunación}

"En comparación con la población general, los pacientes con cáncer en general son más susceptibles a las infecciones prevenibles con vacunas, ya sea por un mayor riesgo debido a la malignidad en sí o al tratamiento inmunosupresor" (29).

Por lo anterior se destaca la importancia de la inmunización en estos pacientes, pues al proporcionar la protección contra infecciones a nivel inmunitario, se disminuye significativamente la incidencia en complicaciones. Existe un riesgo alto de infección en el paciente inmunosuprimido que, a través de la vacunación, se puede llegar a prevenir. Debido a la condición especial que presentan estos pacientes hay que tener en cuenta algunas recomendaciones a la hora de la aplicación de estas $(34,35)$. (Tabla 3). Se recomienda la inmunización de las mascotas (perros y gatos), de la familia y del paciente (36).

\section{Recomendación 2.7 - catéter venoso central}

Se define como: "Dispositivo vascular que se inserta en forma percutánea, a través de una vena central de mayor calibre (vena subclavia, yugular o femoral)" $(37,38)$. Entendemos por "acceso venoso central", el abordaje de una vena profunda, localizada centralmente en el organismo. Aunque su inserción puede ser periférica, la localización de la punta del catéter acaba siendo central. Estos catéteres tienen grandes ventajas, sobre todo a largo plazo, ya que disminuyen la ansiedad del paciente asociada con las punciones venosas repetidas, o que estos tengan un acceso venoso periférico limitado (Tabla 4) (39).

Una de las tres causas de infecciones se relaciona con el uso de dispositivos vasculares. Si bien son imprescindibles para el tratamiento del paciente, representan una puerta abierta a las infecciones, dando pie para la bacteriemia, complicación muy grave del uso de accesos venosos (40).

\section{Cuidados del CVC}

- Seleccionar el catéter, técnica y lugar de inserción con menor riesgo de complicaciones, dependiendo del tipo y duración de la terapia a administrar.

- Antisepsia de la piel e higiene las manos.

- Desinfectar la piel limpia con un antiséptico apropiado antes de inserción del catéter y durante los cambios de apósitos.

- Técnica estéril al manipular el CVC.

- Notificar signos y síntomas de infección.

- Educación del paciente y del cuidador (40).

Tabla 3. Parámetros de inmunización del paciente oncológico.

\begin{tabular}{lll} 
TIPO DE VACUNA & TIEMPO RECOMENDADO & PATÓGENO \\
\hline INACTIVADO & 2 semanas antes de iniciar & Influenza. \\
& la quimioterapia o terapia & Hepatitis A - B. \\
& inmunosupresora. & DPT. \\
& & Meningococo. \\
& & Vph. \\
& & Neumococo. \\
\hline VIRUS ATENUADO & Mínimo 4 semanas antes de la & Varicela/zoster. \\
& terapia inmunosupresora. & Triple viral (SRP).
\end{tabular}

Fuente: Lavergne, M.; Ghannoum; K Weiss, J Roy and C Béliveau, Successful prevention of respiratory syncytial virus nosocomial transmission following an enhanced seasonal infection control program, 2011. Disponible en: http://web.b.ebscohost.com.aure.unab.edu.co/ehost/pdfviewer/pdfviewer?vid=1\&sid=246b849a-c196-4639a1dc-3bc18b4f9ec1\%40sessionmgr102 (36). 
Tabla 4. Tipos de CVC

\begin{tabular}{|c|c|c|c|}
\hline CATÉTER & BENEFICIOS & & DESVENTAJAS \\
\hline No tunelizado & $\begin{array}{l}\text { Vía percutánea en las } \\
\text { venas subclavia, yugular y } \\
\text { femoral. }\end{array}$ & $\begin{array}{l}\text { Administración de fluidos } \\
\text { y de la realización de la } \\
\text { quimioterapia, toma de } \\
\text { muestras de sangre durante } \\
\text { la terapia y no requiere } \\
\text { quirófano. }\end{array}$ & $\begin{array}{l}\text { Urgencias, uso menor a } \\
14 \text { días, mayor riesgo de } \\
\text { complicación. }\end{array}$ \\
\hline Tunelizado o hickman & $\begin{array}{l}\text { En el tórax a través de } \\
\text { una vena grande, con el } \\
\text { objetivo de llegar a las } \\
\text { zonas cercanas del corazón. }\end{array}$ & $\begin{array}{l}\text { Tratamiento para la } \\
\text { quimioterapia. }\end{array}$ & $\begin{array}{l}\text { Infección o sangrado en el } \\
\text { lugar de entrada o salida } \\
\text { o en el túnel subcutáneo, } \\
\text { coágulos de sangre en } \\
\text { o alrededor del catéter, } \\
\text { colapso pulmonar durante } \\
\text { la inserción y la oclusión } \\
\text { del catéter. }\end{array}$ \\
\hline $\begin{array}{l}\text { Implantados o puerto } \\
\text { implantado o "Port-a-Cath" }\end{array}$ & $\begin{array}{l}\text { Reservorio creado } \\
\text { quirúrgicamente y un } \\
\text { catéter tunelizado bajo } \\
\text { la piel hasta la vena cava } \\
\text { superior. }\end{array}$ & $\begin{array}{l}\text { Acceso sencillo, } \\
\text { seguro y permanente al } \\
\text { sistema vascular Menos } \\
\text { interferencias con las } \\
\text { actividades de la vida } \\
\text { diaria, menor impacto en la } \\
\text { autoimagen. }\end{array}$ & $\begin{array}{l}\text { Infección del sitio del } \\
\text { puerto o catéter, coágulos } \\
\text { de sangre, extravasaciones, } \\
\text { colapso pulmonar durante } \\
\text { la inserción y la oclusión } \\
\text { del catéter. }\end{array}$ \\
\hline $\begin{array}{l}\text { Catéteres Centrales de } \\
\text { Inserción Periférica (PICC) }\end{array}$ & $\begin{array}{l}\text { Se introduce en las venas } \\
\text { del brazo superior y se } \\
\text { enrosca en las venas más } \\
\text { grandes en el pecho. }\end{array}$ & $\begin{array}{l}\text { Facilitan la administración } \\
\text { de quimioterapia y la } \\
\text { terapia de apoyo en el } \\
\text { entorno del cáncer. }\end{array}$ & $\begin{array}{l}\text { infección en el sitio de } \\
\text { salida, coágulos de sangre } \\
\text { en el catéter o alrededor } \\
\text { de este; y la oclusión del } \\
\text { catéter. }\end{array}$ \\
\hline
\end{tabular}

Fuente: García Y. Papel de enfermería en la vigilancia, prevención y control de las infecciones nosocomiales. 2012. Disponible en: http://zaguan.unizar.es/record/7227/files/TAZ-TFG- 2012- 066.pdf (40).

\section{Recomendación 2.8 - catéter urinario}

"La infección del tracto urinario (ITU) asociada a catéter vesical es aquella que se presenta en algún momento de los siete días posteriores a la colocación de la sonda de Foley, y que se confirma mediante urocultivo positivo" (41). El principal agente etiológico Escherichia coli, para la cual se ha reportado una elevada resistencia a antibióticos del grupo de b-lactámicos y quinolonas. Los pacientes con cinco días, o más, de colocación de la sonda de Foley presentaron prácticamente cinco veces el riesgo de infección de vías urinarias (41).

\section{Recomendaciones para la prevención de infecciones de las vías urinarias (IVU) con sondas urinarias}

- Uso de técnica estéril para la inserción y manejo de la sonda, de ser posible con circuito cerrado.

- Evaluación diaria de la necesidad de la sonda. De no ser necesaria, proceder al retiro.

- Mantener el sistema de drenaje por debajo del nivel de la vejiga, evitando el contacto con el piso.

- Vigilar, informar y documentar sintomatología correspondiente a una IVU (42). 


\section{Conclusiones}

Según lo encontrado en la revisión de literatura, se hace alusión a la importancia de vigilar, identificar y prevenir de manera oportuna aquellas causas más comunes en el desarrollo de infecciones en el paciente oncológico. A su vez, utilizar diferentes métodos para el control de las mismas, debido a que el paciente oncológico es un individuo que tiene mayor riesgo de infección por estar expuesto a múltiples variables como la enfermedad de base, el tipo de terapia a la que es sometido, su estado nutricional (el cual es la recomendación específica más importante en esta población por las necesidades especiales que se presentan en este aspecto), los procedimientos invasivos, internaciones frecuentes y prolongadas, la posibilidad de colonización con gérmenes multirresistentes del área hospitalaria, el uso de profilaxis antibiótica y tratamientos empíricos, o por la combinación de todos estos factores (42).

Dentro de las medidas y los programas de control de infecciones en los pacientes oncológicos se concluye que tienen componentes en los cuales se abarcan precauciones estándar, precauciones de aislamiento, saneamiento ambiental y vigilancia, siendo la higiene de manos la medida general más importante para reducir la transmisión de microorganismos de una persona a otra o de una parte del cuerpo a otra. Se hace énfasis en la educación del personal de salud y del familiar para disminuir ese riesgo de infección, haciéndolos partícipes del cuidado basado en la evidencia.

\section{Conflicto de intereses}

Los autores manifiestan no tener conflictos de intereses.

\section{Referencias}

1. MinSalud establece reporte para registrar información de pacientes con cáncer. Boletín de Prensa, No 038, Febrero 4 de 2014. Disponible en: http:/www. minsalud.gov.co/Paginas/MinSalud-establecereporte-para-registrar-informaci $\% \mathrm{C} 3 \% \mathrm{~B} 3 n$-depacientes-con-c $\% \mathrm{C} 3 \% \mathrm{~A} 1$ ncer.aspx

2. Instituto nacional del cáncer. Estatificación del cáncer [sede web] 03 de mayo de 2014. Disponible en: http:// www.cancer.gov/espanol/cancer/que-es

3. Organización Mundial de la Salud. Cáncer, sitio de prensa [sede web]. Disponible en: http://www.who. int/mediacentre/factsheets/fs297/es

4. Rodríguez MA, et al. Comité de Control y Prevención de Infecciones asociadas a la Asistencia Sanitaria.
Sección de Epidemiología Hospitalaria [sede web]. Diciembre 2 de 2012. Disponible en: http:// instituciones.sld.cu/seccioneh/

5. Ministerio de salud y protección social. Modelo de cuidado al paciente con cáncer. INSTITUTO NACIONAL DE CANCEROLOGÍA ESE. 2015;0( 978-958-58832-4-6):

6. Whoint. Organización Mundial de la Salud. [sede web]. Disponible en: http://www.who.int/mediacentre/ factsheets/fs297/es/ [Accessed 28 March 2018].

7. Velázquez Brizuela IE, Aranda Gama J, Camacho Cortes JL, Ortiz GG. Epidemiología de infecciones nosocomiales en el Instituto Jalisciense de Cancerología. Disponible en: http://www.bvs.sld.cu/ revistas/spu/vol39_1_13/spu03113.htm

8. Organización mundial de la Salud (OMS), Organización Panamericana de la Salud. Centro de prensa. Cáncer. Disponible en: http:/www.paho. org/hq/index.php?option=com_content\&view=art icle $\&$ id $=292 \% 3$ Acancer $\&$ catid $\overline{=}=1866 \% 3$ Acancerhome\&Itemid $=386 \&$ lang $=$ es

9. Ortiz Luis SR. Educar para mejorar en la prevención de infecciones de vías urinarias en pacientes con sonda vesical instalada. Rev Enferm IMSS 2017; 25(2)

10. MinSalud establece reporte para registrar información de pacientes con cáncer. Boletín de Prensa, No 038, Febrero 4 de 2014. Disponible en: http://www. minsalud.gov.co/Paginas/MinSalud-establecereporte-para-registrar-informaci $\% \mathrm{C} 3 \% \mathrm{~B} 3 \mathrm{n}$-depacientes-con-c $\% \mathrm{C} 3 \% \mathrm{~A} 1 \mathrm{ncer} . \mathrm{aspx}$

11. Maldonado Regalado MS. Infecciones en el paciente oncológico. REV ESP PEDIATR 2013; 69(3): 140154.

12. Instituto Nacional de salud. Marzo del 2011. Disponible en: http://www.ins.gov.co/iaas/Paginas/ que-son-las-iaas.aspx

13. Organización mundial de la salud [sede web]. Una atención limpia es una atención más segura. 2005 Disponible en: http://www.who.int/gpsc/background/ es/

14. Magnus TP, Marra AR, Sampaio Camargo TZ, Victor E, Sodré da Costa LS, Cardoso V, et al. Measuring hand hygiene compliance rates in different special care settings: a comparative study of methodologies; International Journal of Infectious Diseases 33 (2015) 205-208.

15. Lupión $\mathrm{C}$, et al. Formación médica continuada: Infección nosocomial. Fundamentos y actuación clínica. Medidas de prevención de la transmisión de microorganismos entre pacientes hospitalizados. Higiene de manos Enferm Infecc Microbiol Clin 2014;32:603-9 - DOI: 10.1016/j.eimc.2014.02.003.

16. Limpieza y desinfección de equipos y superficies ambientales en instituciones prestadoras de servicios 
de salud; Secretaría distrital de salud dirección de salud pública, 2011

17. Bhalchandra R, Chandy M, V Ramanan, A Mahajan, J Soundaranayagam, S Garai, et al. Role of water quality assessments in hospital infection control: Experience from a new oncology center in eastern India; Indian Journal of Pathology and Microbiology 57(2014) 435438.

18. MANUAL DE BIOSEGURIDAD UNIVERSIDAD INDUSTRIAL DE SANTANDER; noviembre 28 de 2012 .Disponible en: https://www.uis.edu.co/ intranet/calidad/documentos/talento\%20humano/ SALUD\%20OCUPACIONAL/MANUALES/ MTH.02.pdf

19. Friedman ND, Sexton MD. Principios generales del control de infecciones. Mayo 2014. Disponible en: http://www.uptodate.com.bases.unab.edu.co/ contents/general-principles-of-infection-control?source $=$ search $\_$result\&search $=$control $+\mathrm{de}+$ infecciones + en + paciente + +oncologico\&selectedTitle $=2 \sim 150$

20. Sokol KA, De la Vega Diaz I, Edmondson Martin K. Masks for prevention of respiratory viruses on the BMT unit: results of a quality initiative, 2016. Disponible en: http://web.b.ebscohost.com.aure.unab.edu.co/ ehost/pdfviewer/pdfviewer?vid=1\&sid=99060d2b290f-4417-afc3-c572f845f3cd\%40sessionmgr104

21. García Lozano T, Egido A, Contel E, Picón M, Martínez M, Aznar E. ¿Es necesario conocer qué trabajadores son portadores de SARM en contacto con enfermos oncológicos?, Rev. esp. quimioter.; 25(2012): 252-255. Disponible en: http://pesquisa. bvsalud.org/portal/resource/es/mdl-23303255

22. Consenso sobre el cuidado del paciente oncológico neutropénico febril: Actualización 2008-2009. Arch. argent. pediatr. [online]. 2010, vol.108, n.2 [citado 2015-06-03], pp. 0-0. Disponible en: $<$ http:// www.scielo.org.ar/scielo.php?script $=$ sci_arttext\&pid=S032500752010000200022\&lng=es\&nrm $=\mathrm{i}$ so $>$.

23. Gómez Candela C, Canales Albendea MA, et al. Intervención nutricional en el paciente oncohematológico; 2012;27(3):669-680.

24. Nee CL, Hanchett S, Wilson J, Cipkala Gaffin J. An Interdisciplinary Approach: The Elimination of the Neutropenic Diet Across the Continuum of Care. Disponible en: https://www.clinicalkey. es.aure.unab.edu.co/\#!/content/journal/1s2.0-S1083879116311211

25. Rubio I, Ferreiro J, Pérez T. Infecciones en el paciente oncológico. Neutropenia febril. 2018. Disponible en: www.elsevier.es/es-revista- gaceta-medicabilbao-316- pdf- S0304485804744662-S300

26. Cancergov National Cancer Institute. [Online]. Disponible en: https://www.cancer.gov/espanol/ cancer/sobrellevar/familia-y-amigos/familiares-acargo-pro-pdq [Accessed 2 April 2018].

27. Achury Saldaña D, Achury Beltrá L. Participación familiar en el cuidado de pacientes críticos: una propuesta de fundamentación teórica. Investig. Enferm. Imagen Desarr. ISSN 0124-205915 (2): 137151, julio-diciembre de 2013.

28. Ariza Heredia EJ, et al. Practical review of immunizations in adult patients with cancer Hum Vaccin Immunother. 2015 Nov 11(11): 2606-2614.Published online 2015 Jun 25. doi: $10.1080 / 21645515.2015 .1062189$

29. López J, Peña AM, Pérez R, Abarca K. Pet ownership in inmunocompromised patients. Rev. chil. infectol. [Internet]. 2013 Feb [citado 2018 Abr 01];30(1):52-62. Disponible en: https://scielo. conicyt.cl/scielo.php?script=sci_arttext\&pid $=$ S071610182013000100009\&lng=es. $\quad \mathrm{http} / / \mathrm{dx}$.doi. org/10.4067/S0716-10182013000100009.

30. VLavergne, MGhannoum, KWeiss, JRoy,CBe'liveau. Successful prevention of respiratory syncytial virus nosocomial transmission following an enhanced seasonal infection control program, 2011. Disponible en: http://web.b.ebscohost.com.aure.unab.edu.co/ ehost/pdfviewer/pdfviewer?vid=1\&sid=246b849ac196-4639-a1dc-3bc18b4f9ec1\%40sessionmgr102

31. Mengíbar F, et al. Aportaciones de enfermería a la prevención de la infección y el manejo de catéteres venenosos. 2014. Disponible en: http:// tauja.ujaen.es/bitstream/10953.1/1340/1/TFG_ MengibarMerino\%2cFrancisco.pdf

32. Oré M. Revisión crítica de la literatura: "Cuidados de enfermería en pacientes oncológicos, que reciban quimioterapia a través de un Port-a-cath o de un PICC" - tesis de grado. 2016. Disponible en: http://eugdspace. eug.es/xmlui/bitstream/handle/123456789/394/ Cuidados\%20de\%20enfermer\%C3\%ADa\%20 en $\% 20$ pacientes $\% 20$ oncol $\% \mathrm{C} 3 \% \mathrm{~B} 3$ gicos $\% 2 \mathrm{c} \% 20$ quimioterapia $\% 20 \mathrm{a} \% 20 \operatorname{trav} \% \mathrm{C} 3 \% \mathrm{~A} 9 \mathrm{~s} \% 20 \mathrm{de} \% 20$ un $\% 20$ Port-a-ath $\% 20$ o $\% 20 \mathrm{de} \% 20$ un $\% 20$ PICC. pdf? sequence $=1 \&$ amp; isAllowed $=y$

33. Estévez L, Rodríguez M, Mejía L. Práctica segura en el cuidado del paciente oncológico con dispositivos vasculares. 2015. Disponible en: http://www. medigraphic.com/pdfs/enfermeriaimss/eim-2015/ eim153i.pdf

34. García Y, et al. Papel de enfermería en la vigilancia, prevención y control de las infecciones nosocomiales. 2012. Disponible en: http://zaguan.unizar.es/ record/7227/files/TAZ-TFG- 2012- 066.pdf

35. Conley SB; Dana-Farber Cancer Institute, Central Line-Associated Bloodstream Infection Prevention: Standardizing Practice Focused on EvidenceBased Guidelines, 2016, disponible en: http:// 
web.b.ebscohost.com.aure.unab.edu.co/ehost/ pdfviewer/pdfviewer?vid=2\&sid=39be25bb-713b4ee9-8aca-d86f97c0716a\%40sessionmgr102

36. Page J, Tremblay M, Nicholas C, James TA. Reducing Oncology Unit Central Line-Associated Bloodstream, 2015. Disponible en: http:// web.b.ebscohost.com.aure.unab.edu.co/ehost/ pdfviewer/pdfviewer?vid=1\&sid=ba6eb7ac-46aa4ee9-b845-cb6fd43c1890\%40sessionmgr104

37. Zakhour R, Chaftari AM, Raad I. Catheterrelated infections in patients with haematological malignancies: novel preventive and therapeutic strategies, 2016. Disponible en: https://www. clinicalkey.es.aure.unab.edu.co/\#!/content/journal/1s2.0-S1473309916302134

38. Galván A, Martínez L, López C. Permanencia de la sonda de Foley asociada a infección urinaria y farmacorresistencia. 2011. Disponible en: http://www. medigraphic.com/pdfs/micro/ei-2011/ei114c.pdf

39. Calil, Cavalcanti K, Soraia G, Silvino, Rosa Z. Acciones y/o intervenciones de enfermería para la prevención de infecciones hospitalarias en pacientes gravemente enfermos: una revisión integrativa. 2014. Disponible en: http://revistas.um.es/eglobal/article/ viewFile/156491/160761
40. Love N; Acute Care at Memorial SloanKettering Cancer Center, New York, NY. Rodrigue D, Catheter-Associated Urinary Tract Infection Prevention in the Oncology Population: An Evidence-Based Approach, 2013. Disponible ne: http://web.b.ebscohost.com.aure.unab.edu.co/ehost/ pdfviewer/pdfviewer?vid=1\&sid=66b2f47c-f6b44712-ae4b-1b40788d9cc6\%40sessionmgr104

41. Subsecretaría de Integración y Desarrollo del Sector Salud, Protocolo para la Estandarización del Cuidado al Paciente con Sonda Vesical, Enfocado a la Prevención de Infecciones Asociadas a la Atención de la Salud. Disponible en: http:/calidad.salud.gob.mx/ site/editorial/docs/protocolo sonda vesical.pdf

42. Tseng H, Moorhead S. The Use of Standardized Terminology to Represent Nursing Knowledge: Nursing Interventions relevant to Safety for Patients with Cancer, 2014. Disponible en: http:// web.a.ebscohost.com.aure.unab.edu.co/ehost/detail/ detail? vid=2\&sid=c0f23f0d-c0b94f72a00817d6f246 $5115 \% 40$ sessionmgr4007\&bdata $=$ Jmxhbmc9ZXMm c210ZT1laG9zdC1saXZl\#AN=24943558\&db=mnh 